\title{
MERSENNE-LUCAS HYBRID NUMBERS
}

\author{
ENGIN ÖZKAN ${ }^{*}$, MINE UYSAL ${ }^{\dagger}$ \\ "Department of Mathematics, Erzincan Binali Yıldırım University, \\ Faculty of Arts and Sciences, 24100 Erzincan, Turkey \\ ${ }^{\dagger}$ Graduate School of Natural and Applied Sciences, \\ Erzincan Binali Yıldırım University, 24100 Erzincan, Turkey. \\ *Corresponding author. E-mail : eozkan@erzincan.edu.tr \\ or eozkanmath@gmail.com
}

\section{DOI:10.20948/mathmontis-2021-52-2}

Summary. We introduce Mersenne-Lucas hybrid numbers. We give the Binet formula, the generating function, the sum, the character, the norm and the vector representation of these numbers. We find some relations among Mersenne-Lucas hybrid numbers, Jacopsthal hybrid numbers, Jacopsthal-Lucas hybrid numbers and Mersenne hybrid numbers. Then we present some important identities such as Cassini identities for Mersenne-Lucas hybrid numbers.

\section{INTRODUCTION}

Number sequences continue to attract the attention of researchers for a long time. Number sequences, especially Fibonacci sequences, find application in many departments of mathematics as well as in other branches of science.

Many researchers have studied Fibonacci numbers and new number sequences created by their generalizations. [1-8].

Koshy [9] written one of the most popular books of Fibonacci and Lucas numbers, and gave numerous recurrence relations, generalizations and applications of Fibonacci and Lucas numbers.

Catarino et. al defined the Mersenne sequence and some identities of the the Mersenne sequence. Later, Saba et.al introduced Mersenne-Lucas nembers and some identity of this sequence.

Later, many researchers studied on hybrid numbers. These researchers developed hybrid numbers by relating them to other number sequences and created other number sequences. [10$18]$.

In this study, Mersenne-Lucas hybrid numbers will be defined by using the hybrid numbers. A generating function and a Binet formula for the Mersenne-Lucas hybrid numbers will be found. Furthermore, the sum, the character, the norm and the vector representation of these numbers will be given. Some relations among Mersenne-Lucas hybrid numbers, Jacopsthal hybrid numbers, Jacopsthal-Lucas hybrid numbers and Mersenne hybrid numbers wil be presented.

2010 Mathematics Subject Classification: 11B37, 11B39, 05A15

Key words and Phrases: Mersenne hybrid numbers, Mersenne-Lucas hybrid numbers, Binet formula, Generating function, Cassini identity, Catalan identity 
Later, we give Cassini identity, Catalan identitiy, Vajda identity, D'ocagne identity and Honsberger identity for Mersenne-Lucas hybrid numbers.

\section{PRELIMINIARIES}

Özdemir introduced the hybrid numbers [13]. The set of hybrid numbers is $K=\{a+b i+c \varepsilon+d h: a, b, c, d \in \mathbb{R}\}$. Let be

$$
z_{1}=a_{1}+b_{1} i+c_{1} \varepsilon+d_{1} h, \quad z_{2}=a_{2}+b_{2} i+c_{2} \varepsilon+d_{2} h
$$

any two hybrid numbers. Then we have the following properties.

- $z_{1}=z_{2} \Leftrightarrow a_{1}=a_{2}, b_{1}=b_{2}, c_{1}=c_{2}$ and $d_{1}=d_{2}$.

- $z_{1}+z_{2}=\left(a_{1}+a_{2}\right)+\left(b_{1}+b_{2}\right) i+\left(c_{1}+c_{2}\right) \varepsilon+\left(d_{1}+d_{2}\right) h$

- $z_{1}-z_{2}=\left(a_{1}-a_{2}\right)+\left(b_{1}-b_{2}\right) i+\left(c_{1}-c_{2}\right) \varepsilon+\left(d_{1}-d_{2}\right) h$

- $\quad k . z_{1}=k a_{1}+k b_{1} i+k c_{1} \varepsilon+k d_{1} h$, where $k \in \mathbb{R}$.

Some basic properties of hybrid counts are given by the following definition.

Definition 2.1. There are the following definitions where $z$ is any hybrid number such as $z=a+b i+c \varepsilon+d h[13]$.

- The conjugate of $z$ is

$$
\bar{z}=a-b i-c \varepsilon-d h
$$

- The character of $z$ is

$$
C(z)=z \bar{z}=a^{2}+(b-c)^{2}-c^{2}-d^{2}=a^{2}+b^{2}-2 b c-d^{2} .
$$

- The norm for $z$ has the form

$$
\|z\|=N(z)=\sqrt{C(z)} .
$$

- $\quad z$ is a spacelike, timelike or lightlike if

$$
C(z)<0, C(z)>0 \text { or } C(z)=0 .
$$

respectively.

- The vector representation for $z$ is

$$
V_{z}=(a, b-c, c, d) \text {. }
$$

- The scalar section of $z$ is

$$
S(z)=a
$$

- The vector section of $z$ is

$$
V(z)=b i+c+d h .
$$

Definition 2.2. The Mersenne numbers $\left\{M_{n}\right\}_{n=0}^{\infty}$ are defined by the following recurrence relations

$$
M_{n+1}=2 M_{n}+1
$$

or

$$
M_{n+2}=3 M_{n+1}-2 M_{n}
$$


with $M_{0}=0$ and $M_{1}=1$ [19].

Definition 2.3. The Binet formula of the Mersenne numbers are defined by the following. [19]

$$
M_{n}=2^{n}-1
$$

Definition 2.4. The Mersenne-Lucas numbers $\left\{m_{n}\right\}_{n=0}^{\infty}$ are defined by the following recurrence

$$
m_{n}=3 m_{n-1}-2 m_{n-2}
$$

with $m_{0}=2$ and $m_{1}=3 .[11]$

Other definition is given by,

$$
m_{n+1}=2 m_{n}-1
$$

Definition 2.5. The Binet formula for Mersenne- Lucas numbers is defined by [20],

$$
m_{n}=2^{n}+1
$$

Definition 2.6. The sum of the Mersenne- Lucas numbers is given by [20],

$$
\sum_{k=0}^{n} m_{k}=2^{n+1}+n
$$

or

$$
\sum_{k=0}^{n} m_{k}=2 m_{n}+n-2
$$

Definition 2.7. The Jacobsthal numbers $\left\{J_{n}\right\}_{n=0}^{\infty}$ are defined by the following recurrence relation,

$$
J_{n+2}=J_{n+1}+2 J_{n}
$$

with $J_{0}=0$ and $J_{1}=1$. [9]

Definition 2.8. The Jacobsthal -Lucas numbers $\left\{j_{n}\right\}_{n=0}^{\infty}$ are given by

$$
j_{n+2}=j_{n+1}+2 j_{n}
$$

with $j_{0}=2$ and $j_{1}=1$. [9]

Definition 2.9. The Mersenne hybrid number, $\left\{M H_{n}\right\}_{n=0}^{\infty}$ is defined as

$$
M H_{n}=M_{n}+i M_{n+1}+\varepsilon M_{n+2}+h M_{n+3}, n \geq 0
$$

where $M_{n}$ is the $n$th Mersenne number. [18]

Now let's give the preliminary information.

\section{MERSENNE-LUCAS HYBRID NUMBERS}

Definition 3.1. Let $n \geq 0$ be integer, Mersenne-Lucas hybrid numbers $\left\{m h_{n}\right\}$ for $n=0, \ldots, \infty$ is defined as,

$$
m h_{n}=m_{n}+i m_{n+1}+\varepsilon m_{n+2}+h m_{n+3}
$$


where, $m_{n}$ is nth Mersenne-Lucas number.

Let us give a few terms of Mersenne-Lucas hybrid numbers in Table 1.

\begin{tabular}{|c|c|}
\hline$n$ & $m h_{n}$ \\
\hline 0 & $2+3 i+5 \varepsilon+9 h$ \\
\hline 1 & $3+5 i+9 \varepsilon+17 h$ \\
\hline 2 & $5+9 i+17 \varepsilon+33 h$ \\
\hline 3 & $9+17 i+33 \varepsilon+65 h$ \\
\hline
\end{tabular}

Table 1: Same values of Mersenne-Lucas hybrid numbers

Theorem 3.2. The Binet formula for $\left\{\mathrm{mh}_{\mathrm{n}}\right\}_{\mathrm{n}=0}^{\infty}$ is defined as,

$$
m h_{n}=2^{\mathrm{n}}(1+2 i+4 \varepsilon+8 h)+(1+i+\varepsilon+h), n \geq 0 .
$$

Proof. From (1) and the Binet formula for Mersenne-Lucas numbers, we have

$$
\begin{aligned}
m h_{n} & =2^{n}+1+i\left(2^{n+1}+1\right)+\varepsilon\left(2^{n+2}+1\right)+h\left(2^{n+3}+1\right) \\
& =2^{n}(1+2 i+4 \varepsilon+8 h)+(1+i+\varepsilon+h)
\end{aligned}
$$

Thus, the proof is complete.

Lemma 3.3. Let $n \geq 0$ be integer, the recurrance relation of Mersenne-Lucas hybrid numbers $\left\{m h_{n}\right\}_{n=0}^{\infty}$ is given as,

$$
m h_{n+2}=3 m h_{n+1}-2 m h_{n}
$$

Proof. From (1), we obtain

$$
\begin{aligned}
& \quad m h_{n+2}=m_{n+2}+i m_{n+3}+\varepsilon m_{n+4}+h m_{n+5} \\
& =\left(3 m_{n+1}-2 m_{n}\right)+i\left(3 m_{n+2}-2 m_{n+1}\right)+\varepsilon\left(3 m_{n+3}-2 m_{n+2}\right)+h\left(3 m_{n+4}-2 m_{n+3}\right) \\
& =3\left(m_{n+1}+i m_{n+2}+\varepsilon m_{n+3}+h m_{n+4}\right)-2\left(m_{n}+i m_{n+1}+\varepsilon m_{n+2}+h m_{n+3}\right) \\
& =3 m h_{n+1}-2 m h_{n}
\end{aligned}
$$

Thus, the desired is obtained.

Theorem 3.4. The generating function for $\left\{\mathrm{mh}_{\mathrm{n}}\right\}_{\mathrm{n}=0}^{\infty}$ is given as follows,

$$
G(t)=\sum_{n=0}^{\infty} m h_{n} t^{n}=\frac{2+3 i+5 \varepsilon+9 h-t(3+4 i+6 \varepsilon+10 h)}{\left(1-3 t+2 t^{2}\right)}
$$


Proof. We have

$$
G(t)=m h_{0}+m h_{1} t+m h_{2} t^{2}+\cdots+m h_{n} t^{n}+\cdots
$$

Let us multiply Equation (2) by $-3 t, 2 t^{2}$ respectively. So, the following equations are obtained.

$$
\begin{aligned}
G(t) & =m h_{0}+m h_{1} t+m h_{2} t^{2}+\cdots+m h_{n} t^{n}+\cdots \\
-3 t G(t) & =-3 t m h_{0}-3 t^{2} m h_{1}-3 t^{3} m h_{2}-\cdots-3 t^{n+1} m h_{n}-\cdots \\
2 t^{2} G(t) & =2 t^{2} m h_{0}+2 t^{3} m h_{1}+2 t^{4} m h_{2}+\cdots+2 t^{n+2} m h_{n}+\cdots
\end{aligned}
$$

If we take the necessary calculations to take advantage of the recurrence relation, we obtain the following equations

$$
\begin{gathered}
G(t)\left(1-3 t+2 t^{2}\right)=m h_{0}+m h_{1} t-3 t m h_{0} \\
G(t)=\frac{2+3 i+5 \varepsilon+9 h+t(3+5 i+9 \varepsilon+17 h)-3 t(2+3 i+5 \varepsilon+9 h)}{\left(1-3 t+2 t^{2}\right)} \\
G(t)=\frac{2+3 i+5 \varepsilon+9 h-t(3+4 i+6 \varepsilon+10 h)}{\left(1-3 t+2 t^{2}\right)}
\end{gathered}
$$

In this case, the desired formula is obtained.

Theorem 3.5. Let $S(t)$ be sum of $\left\{\mathrm{mh}_{\mathrm{n}}\right\}_{\mathrm{n}=0}^{\infty}$. Then we have,

$$
S(t)=\sum_{\mathrm{k}=0}^{\mathrm{n}} m h_{k}=2 m h_{n}+n-2+i(n-3)+\varepsilon(n-5)+h(n-9)
$$

Proof. We have,

$$
S(t)=m h_{0}+m h_{1}+m h_{2}+\cdots+m h_{n}
$$

From (2.1), we have

$$
\begin{gathered}
S(t)=m_{0}+i m_{1}+\varepsilon m_{2}+h m_{3}+m_{1}+i m_{2}+\varepsilon m_{3}+h m_{4}+\cdots+m_{n} \\
+i m_{n+1}+\varepsilon m_{n+2}+h m_{n+3} \\
=\left(m_{0}+m_{1}+\cdots+m_{n}\right)+i\left(m_{1}+\cdots+m_{n+1}\right) \\
+\varepsilon\left(m_{2}+\cdots+m_{n+2}\right)+h\left(m_{3}+\cdots+m_{n+3}\right)
\end{gathered}
$$

From the sum of Mersenne numbers, we get the following

$$
\begin{aligned}
= & \left(2 m_{n}+n-2\right)+i\left(2 m_{n+1}+n-1-2\right)+\varepsilon\left(2 m_{n+2}+n-2-3\right) \\
& +h\left(2 m_{n+3}+n+1-2-3-5\right) \\
= & 2\left(m_{n}+i m_{n+1}+\varepsilon m_{n+2}+h m_{n+3}\right)+n-2+i(n-3)+\varepsilon(n-5)+h(n-9) \\
= & 2 m h_{n}+n-2+i(n-3)+\varepsilon(n-5)+h(n-9)
\end{aligned}
$$

Thus, proof is complete.

Theorem 3.6. The character of $\left\{\mathrm{mh}_{\mathrm{n}}\right\}_{\mathrm{n}=0}^{\infty}$ is 


$$
C\left(m h_{n}\right)=-35 m_{n}^{2}-54 m^{2}{ }_{n+1}+88 m_{n} m_{n+1}, \quad n \geq 0
$$

where $m_{n}$ is the $n t h$ Mersenne-Lucas number.

Proof. From definition character of hybrid numbers, we get

$$
\begin{aligned}
C\left(m h_{n}\right)=m h_{n} \overline{m h_{n}}= & \left(m_{n}+i m_{n+1}+\varepsilon m_{n+2}+h m_{n+3}\right)\left(m_{n}-i m_{n+1}\right. \\
& \left.-\varepsilon m_{n+2}-h m_{n+3}\right) \\
= & m^{2}{ }_{n}+m^{2}{ }_{n+1}-2 m_{n+1} m_{n+2}-m^{2}{ }_{n+3}
\end{aligned}
$$

Using reccurance relation of Mersenne numbers are the following,

$$
m_{n+2}=3 m_{n+1}-2 m_{n}
$$

and

$$
\begin{aligned}
m_{n+3} & =3 m_{n+2}-2 m_{n+1} \\
& =3\left(3 m_{n+1}-2 m_{n}\right)-2 m_{n+1} \\
& =7 m_{n+1}-6 m_{n}
\end{aligned}
$$

Then, we have,

$$
\begin{aligned}
C\left(m h_{n}\right) & =m^{2}{ }_{n}+m^{2}{ }_{n+1}-2 m_{n+1}\left(3 m_{n+1}-2 m_{n}\right)-\left(7 m_{n+1}-6 m_{n}\right)^{2} \\
& =m_{n}^{2}+m_{n+1}^{2}-6 m^{2}{ }_{n+1}+4 m_{n+1} m_{n}-49 m^{2}{ }_{n+1}+84 m_{n+1} m_{n}-36 m^{2}{ }_{n} \\
& =-35 m^{2}{ }_{n}-54 m^{2}{ }_{n+1}+88 m_{n+1} m_{n}
\end{aligned}
$$

Thus, the proof is complete.

Theorem 3.7. For any $n \geq 0$, Mersenne-Lucas hybrid number is spacelike.

Proof. From the definitions of Character and Binet formula of Mersenne-Lucas numbers, we obtain that,

$$
\begin{aligned}
C\left(m h_{n}\right) & =-35 m^{2}{ }_{n}-54 m^{2}{ }_{n+1}+88 m_{n+1} m_{n} \\
& =-35\left(2^{n}+1\right)^{2}-54\left(2^{n+1}+1\right)^{2}+88\left(2^{n+1}+1\right)\left(2^{n}+1\right) \\
& =-75.2^{2 n}-22.2^{n}-1
\end{aligned}
$$

Since $C\left(m h_{n}\right)<0$ for any $n \geq 0$, Mersenne-Lucas hybrid number is spacelike.

Theorem 3.8. The vector representation of Mersenne-Lucas Hybrid numbers provide the following identities.

$$
V_{m h_{n}}=3 V_{m h_{n+1}}-2 V_{m h_{n+2}}
$$

Proof. By using definition the vector representation of Mersenne in Definition 1.1., we get

$$
\begin{gathered}
3 V_{m h_{n+1}}-2 V_{m h_{n+2}} \\
=\left(3 m_{n+1}, 3 m_{n+2}-3 m_{n+3}, 3 m_{n+4}, 3 m_{n+5}\right)-\left(2 m_{n+2}, 2 m_{n+3}-2 m_{n+4}, 2 m_{n+5}, 2 m_{n+6}\right)
\end{gathered}
$$




$$
\begin{aligned}
= & \left(3 m_{n+1}-2 m_{n+2}, 3 m_{n+2}-2 m_{n+3}-3 m_{n+3}-2 m_{n+4}, 3 m_{n+4}-2 m_{n+5}, 3 m_{n+5}\right. \\
& \left.-2 m_{n+6}\right)
\end{aligned}
$$

From the reccurence relation of Mersenne numbers,

$=\left(m_{n}, m_{n+1}-m_{n+2}, m_{n+3}, m_{n+4}\right)$

Thus,

$$
V_{m h_{n}}=\left(m_{n}, m_{n+1}-m_{n+2}, m_{n+3}, m_{n+4}\right)=3 V_{m h_{n+1}}-2 V_{m h_{n+2}}
$$

is obtained.

Definition 3.9. Let $N\left(m h_{n}\right)$ be norm of the Mersenne-Lucas hybrid numbers. Then we have,

$$
N\left(m h_{n}\right)=\sqrt{C\left(m h_{n}\right)}=\sqrt{-35 m^{2}{ }_{n}-54 m^{2}{ }_{n+1}+88 m_{n+1} m_{n}} .
$$

Theorem 3.10. We have the following properties,

$$
\begin{aligned}
& \text { i) } m h_{n}+m h_{n+1}=3.2^{n}(1+2 i+4 \varepsilon+8 h)+2(1+i+\varepsilon+h) \\
& \text { ii) } m h_{n+1}=2 m h_{n}-(1+i+\varepsilon+h)
\end{aligned}
$$

Proof. From (2.1), we have

i) $m h_{n}+m h_{n+1}$

$$
\begin{aligned}
= & \left(m_{n}+i m_{n+1}+\varepsilon m_{n+2}+h m_{n+3}\right)+\left(m_{n+1}+i m_{n+2}+\varepsilon m_{n+3}+h m_{n+4}\right) \\
= & 2^{n}+1+i\left(2^{n+1}+1\right)+\varepsilon\left(2^{n+2}+1\right)+h\left(2^{n+3}+1\right) \\
& +\left(2^{n+1}+1\right)+i\left(2^{n+2}+1\right)+\varepsilon\left(2^{n+3}+1\right)+h\left(2^{n+4}+1\right) \\
= & 3.2^{n}(1+2 i+4 \varepsilon+8 h)+2(1+i+\varepsilon+h)
\end{aligned}
$$

So, the proof is complete.

ii) $m h_{n+1}=m_{n+1}+i m_{n+2}+\varepsilon m_{n+3}+h m_{n+4}$

From reccurance relation of Mersenne-Lucas number, we have

$$
\begin{aligned}
& =2 m_{n}-1+i\left(2 m_{n+1}-1\right)+\varepsilon\left(2 m_{n+2}-1\right)+h\left(2 m_{n+3}-1\right) \\
& =2\left(m_{n}+i m_{n+1}+\varepsilon m_{n+2}+h m_{n+3}\right)-(1+i+\varepsilon+h) \\
& =2 m h_{n}-(1+i+\varepsilon+h)
\end{aligned}
$$

Thus, the proof is complete.

Lemma 3.11. We have the following relations
i) $m_{n}=\left\{\begin{array}{cl}3 J_{n}, & \text { if } n \text { is even } \\ 3 J_{n}+2, & \text { if } n \text { is odd }\end{array}\right.$
ii) $m_{n}=\left\{\begin{array}{cl}j_{n}, & \text { if } n \text { is even } \\ j_{n}+2, & \text { if } n \text { is odd }\end{array}\right.$ 
Proof. The proof is easily shown by induction over $n$.

Theorem 3.12. By the following identities between Mersenne-Lucas hybrid number, Jacobshtal hyrid numbers and Jacobshtal-Lucas hyrid numbers are provided.

$$
\begin{aligned}
& \text { i) } m h_{n}+m h_{n+1}=3\left(J H_{n}+J H_{n+1}\right)+2(1+i+\varepsilon+h) \\
& \text { ii) } m h_{n}+m h_{n+1}=j H_{n}+j H_{n+1}+2+3 i+5 \varepsilon+5 h
\end{aligned}
$$

Proof. $i$ ) Let's $n$ is even. From Lemma 2.2., we obtain

$$
\begin{aligned}
m h_{n} & =m_{n}+i m_{n+1}+\varepsilon m_{n+2}+h m_{n+3} \\
& =3 J_{n}+\mathrm{i}\left(3 J_{n+1}+2\right)+\varepsilon\left(3 J_{n+2}\right)+h\left(3 J_{n+3}+2\right) \\
& =3 J_{n}+i 3 J_{n+1}+\varepsilon 3 J_{n+2}+h 3 J_{n+3}+2 h+2 i \\
& =3\left(J_{n}+\mathrm{i} J_{n+1}+\varepsilon J_{n+2}+\mathrm{h} J_{n+3}\right)+2 h+2 i
\end{aligned}
$$

From the definition of Jacobshtal hyrid numbers, we have

$$
\begin{aligned}
m h_{n} & =3 J H_{n}+2 h+2 i \\
m h_{n+1} & =m_{n+1}+i m_{n+2}+\varepsilon m_{n+3}+h m_{n+4} \\
& =\left(3 J_{n+1}+2\right)+i\left(3 J_{n+2}\right)+\varepsilon\left(3 J_{n+3}+2\right)+h\left(3 J_{n+4}\right) \\
& =3\left(J_{n+1}+i J_{n+2}+\varepsilon J_{n+3}+h J_{n+4}\right)+2+2 \varepsilon
\end{aligned}
$$

From the definition of Jacobshtal hybrid numbers, we get

$$
=3 J H_{n+1}+2+2 \varepsilon
$$

So, we obtain the following equation,

$$
\begin{aligned}
m h_{n}+m h_{n+1} & =3 J H_{n}+2 \mathrm{~h}+2 \mathrm{i}+3 J H_{n+1}+2+2 \varepsilon \\
& =3\left(J H_{n}+J H_{n+1}\right)+2(1+i+\varepsilon+h)
\end{aligned}
$$

Similarly, it is shown in the state of the $n$.

ii) Let's $n$ is even. From Lemma 2.2, we get

$$
\begin{aligned}
m h_{n} & =m_{n}+i m_{n+1}+\varepsilon m_{n+2}+h m_{n+3} \\
& =\left(j_{n}+2\right)+i\left(j_{n+1}\right)+\varepsilon\left(j_{n+2}+2\right)+h\left(j_{n+3}\right) \\
& =j_{n}+i j_{n+1}+\varepsilon j_{n+2}+h j_{n+3}+2+i+2 \varepsilon+3 h
\end{aligned}
$$

From the definition of Jacobshtal-Lucas hybrid numbers, we have

$$
\begin{aligned}
m h_{n+1} & =m_{n+1}+i m_{n+2}+\varepsilon m_{n+3}+h m_{n+4} \\
& =j_{n+1}++i\left(j_{n+2}+2\right)+\varepsilon\left(j_{n+3}\right)+h\left(j_{n+4}+2\right) \\
& =j_{n+1}+i j_{n+2}+\varepsilon j_{n+3}+h j_{n+4}+2 i+3 \varepsilon+2 h \\
& =j H_{n+1}+2 i+3 \varepsilon+2 h
\end{aligned}
$$


So, we get

$$
\begin{aligned}
m h_{n}+m h_{n+1} & =j H_{n}+2+i+2 \varepsilon+3 h+j H_{n+1}+2 \mathrm{i}+3 \varepsilon+2 h \\
& =j H_{n}+j H_{n+1}+2+3 i+5 \varepsilon+5 h
\end{aligned}
$$

Similarly, it is shown in the state of the $n$.

Thus the proof is complete.

Theorem 3.13. There is a relationship between Mersenne-Lucas hybrid numbers and Mersenne hyrid numbers

$$
m h_{n}=2 M H_{n+1}-3 M H_{n}
$$

Proof. Let's use the Binet formula for the right hand side of the equation. Then we get

$$
\begin{aligned}
2 M H_{n+1}-3 M H_{n} & =2.2^{n+1}(1+2 i+4 \varepsilon+8 h)-2(1+i+\varepsilon+h) \\
-3 . & 2^{n}(1+2 i+4 \varepsilon+8 h)+3(1+i+\varepsilon+h) \\
& =2^{n}(1+2 i+4 \varepsilon+8 h)+(1+i+\varepsilon+h)=m h_{n}
\end{aligned}
$$

Thus,

$$
m h_{n}=2 M H_{n+1}-3 M H_{n}
$$

is obtained.

Theorem 3.14. For $n \geq 0$, the following equations are provided

$$
\begin{aligned}
\text { i) } S\left(m h_{n}\right) & =\left\{\begin{array}{cc}
3 J_{n}, & \text { if } n \text { is even } \\
3 J_{n}+2, & \text { if } n \text { is odd }
\end{array}\right. \\
\text { ii) } S\left(m h_{n}\right) & =\left\{\begin{array}{cc}
j_{n}, & \text { if } n \text { is even } \\
j_{n}+2, & \text { if } n \text { is odd }
\end{array}\right.
\end{aligned}
$$

where $S\left(m h_{n}\right)$ are the scalar parts of Mersenne-Lucas numbers.

Proof. i). By using the definition of the scalar parts of Mersenne in Definition 1.1., we have

$$
S\left(m h_{n}\right)=m_{n}
$$

From Lemma 2.2., if $n$ is even then we get

$$
S\left(m h_{n}\right)=m_{n}=3 J_{n}
$$

If $n$ is odd, then we have

$$
S\left(m h_{n}\right)=m_{n}=3 J_{n}+2
$$

ii). From the definition of the scalar parts of Mersenne, we get

$$
S\left(m h_{n}\right)=m_{n}
$$

From Lemma 2.2., if $n$ is even, then we get

$$
S\left(m h_{n}\right)=m_{n}=\mathrm{j}_{n}
$$


If $n$ is odd, then we have

$$
S\left(m h_{n}\right)=m_{n}=\mathrm{j}_{n}+2
$$

Thus, the proof is obtained.

We will now give some important identities regarding the Mersenne-Lucas hybrid numbers.

Theorem 3.15. Cassini identity of Mersenne-Lucas hybrid numbers for $n>0$ as follows:

$$
m h_{n-1} m h_{n+1}-m h^{2}{ }_{n}=2^{n-1}(13+21 i+11 \varepsilon+3 h)
$$

Proof. For proof, let's write the left side of the equality by using the binet formula, we get

$$
\begin{aligned}
& m h_{(n-1)} m h_{(n+1)}-m h^{2}{ }_{n} \\
= & 2^{n-1}(1+2 i+4 \varepsilon+8 h)(1+i+\varepsilon+h)+2^{n+1}(1+i+\varepsilon+h)(1+2 i+4 \varepsilon+8 h) \\
& \quad-2^{n}(1+2 i+4 \varepsilon+8 h)(1+i+\varepsilon+h)-2^{n}(1+i+\varepsilon+h)(1+2 i+4 \varepsilon+8 h) \\
= & 2^{n-1}(13-3 \mathrm{i}+3 \varepsilon+11 \mathrm{~h})+2^{n+1}(13+9 \mathrm{i}+7 \varepsilon+7 h)-2^{n}(13-3 \mathrm{i}+3 \varepsilon+11 \mathrm{~h}) \\
\quad & \quad-2^{n}(13+9 \mathrm{i}+7 \varepsilon+7 \mathrm{~h}) \\
= & -2^{n-1}(13-3 i+3 \varepsilon+11 h)+2^{n}(13+9 i+7 \varepsilon+7 h) \\
= & 2^{n-1}(13+21 i+11 \varepsilon+3 h)
\end{aligned}
$$

Thus the proof is obtained.

Theorem 3.16. Catalan identity of Mersenne-Lucas hybrid numbers for $n, r \geq 0$ as follows:

$$
\begin{aligned}
m h_{n-r} m h_{n+r}-m h^{2}{ }_{n}= & 2^{n-\mathrm{r}}\left(1-2^{\mathrm{r}}\right)(13-3 i+3 \varepsilon+11 h \\
& \left.-2^{\mathrm{r}}(13+9 i+7 \varepsilon+7 h)\right)
\end{aligned}
$$

Proof.

$$
\begin{aligned}
& \quad m h_{n-r} m h_{n+r}-m h^{2}{ }_{n} \\
& =2^{n-\mathrm{r}}(1+2 i+4 \varepsilon+8 h)(1+i+\varepsilon+h)+2^{n+\mathrm{r}}(1+i+\varepsilon+h)(1+2 i+4 \varepsilon+8 h) \\
& \quad-2^{n}(1+2 i+4 \varepsilon+8 h)(1+i+\varepsilon+h)-2^{n}(1+i+\varepsilon+h)(1+2 i+4 \varepsilon+8 h) \\
& =2^{n-\mathrm{r}}(13-3 i+3 \varepsilon+11 h)+2^{n+\mathrm{r}}(13+9 i+7 \varepsilon+7 h)-2^{n}(13-3 i+3 \varepsilon+11 \mathrm{~h}) \\
& \quad-2^{n}(13+9 i+7 \varepsilon+7 h) \\
& =2^{n-\mathrm{r}}(13-3 i+3 \varepsilon+11 h)\left(1-2^{\mathrm{r}}\right)+2^{\mathrm{n}}(13+9 i+7 \varepsilon+7 h)\left(2^{\mathrm{r}}-1\right) \\
& =2^{n-\mathrm{r}}\left(1-2^{\mathrm{r}}\right)\left[13-3 i+3 \varepsilon+11 h-2^{\mathrm{r}}(13+9 i+7 \varepsilon+7 h)\right]
\end{aligned}
$$

Thus the proof is obtained.

If we write $r=1$, then we get the Cassini identity.

Theorem 3.17. Vajda identity of Mersenne-Lucas hybrid numbers for $n, m, r \geq 0$ as follows:

$$
\begin{gathered}
m h_{n+r} m h_{n+k}-m h_{n} m h_{n+r+k} \\
=2^{2 n-\mathrm{r}}\left(2^{r}-1\right)\left[\left(13-3 i+3 \varepsilon+11 h-2^{\mathrm{k}}(13+9 i+7 \varepsilon+7 h)\right]\right.
\end{gathered}
$$


Proof. For proof, let's write the left side of the equality by using the Binet formula, we get

$$
\begin{aligned}
& m h_{n+r} m h_{n+k}-m h_{n} m h_{n+r+k} \\
& =2^{n+\mathrm{r}}(13-3 i+3 \varepsilon+11 h)+2^{n+k}(13+9 i+7 \varepsilon+7 h)-2^{n}(13-3 i+3 \varepsilon+11 h) \\
& \quad \quad-2^{n+r+k}(13+9 i+7 \varepsilon+7 h) \\
& =2^{n}(13-3 i+3 \varepsilon+11 h)\left(2^{\mathrm{r}}-1\right)-2^{n+k}(13+9 i+7 \varepsilon+7 h)\left(2^{\mathrm{r}}-1\right) \\
& =2^{n}\left(2^{\mathrm{r}}-1\right)\left((13-3 i+3 \varepsilon+11 h)-2^{\mathrm{k}}(13+9 i+7 \varepsilon+7 h)\right)
\end{aligned}
$$

Thus, the desired expression is obtained.

Theorem 3.18. D'ocagne identity of Mersenne-Lucas hybrid numbers for $n, m \geq 0$ as follows:

$$
m h_{m} m h_{n+1}-m h_{n} m h_{m+1}=2^{n-m}(13+2 i+11 \varepsilon+3 h)
$$

Proof. For proof, let's write the left side of the equality by using the Binet formula,then we get

$$
\begin{aligned}
& \quad m h_{m} m h_{n+1}-m h_{n} m h_{m+1} \\
& \left.=2^{m}(1+2 i+4 \varepsilon+8 h)+(1+i+\varepsilon+h)\right]\left[2^{n+1}(1+2 i+4 \varepsilon+8 h)+(1+i+\varepsilon+h)\right] \\
& \quad-\left[2^{n}(1+2 i+4 \varepsilon+8 h)+(1+i+\varepsilon+h)\right]\left[2^{m+1}(1+2 i+4 \varepsilon+8 h)\right. \\
& =2^{m}(13-3 i+3 \varepsilon+11 h)+2^{n+1}(13+9 i+7 \varepsilon+7 h)-2^{n}(13-3 i+3 \varepsilon+11 h) \\
& \quad-2^{m+1}(13+9 i+7 \varepsilon+7 h) \\
& =(13-3 i+3 \varepsilon+11 h)\left(2^{m}-2^{n}\right)+(13+9 i+7 \varepsilon+7 h)\left(2^{n+1}-2^{m+1}\right) \\
& =2^{n-m}(13+2 i+11 \varepsilon+3 h)
\end{aligned}
$$

Thus, the proof is obtained.

Theorem 3.19. Honsberger identity of Mersenne-Lucas hybrid numbers for $n, m \geq 0$ as follows:

$$
\begin{gathered}
m h_{n} m h_{m}+m h_{n+1} m h_{m+1}=\left(2^{n+m} 385+2^{n} 39+2^{m} 39+6\right) \\
+\left(2^{n+m} 20-2^{n} 9+2^{m} 27+4\right) i+\left(2^{n+m} 40+2^{n} 9+2^{m} 21+4\right) \varepsilon \\
+\left(2^{n+m} 80+2^{n} 33+2^{m} 21+4\right) h
\end{gathered}
$$

Proof. For proof, let's write the left side of the equality by using the Binet formula, then we get

$$
\begin{aligned}
& \quad m h_{n} m h_{m}+m h_{n+1} m h_{m+1} \\
& \left.=2^{n}(1+2 i+4 \varepsilon+8 h)+(1+i+\varepsilon+h)\right]\left[2^{m}(1+2 i+4 \varepsilon+8 h)\right. \\
& +(1+i+\varepsilon+h)]+\left[2^{n+1}(1+2 i+4 \varepsilon+8 h)+(1+i+\varepsilon+h)\right] \\
& {\left[2^{m+1}(1+2 i+4 \varepsilon+8 h)+(1+i+\varepsilon+h)\right]} \\
& =\left(2^{n+m} 5(77+4 i+8 \varepsilon+16 h)\right)+2^{n} 3(13-3 i+3 \varepsilon+11 h) \\
& +2^{m} 3(13+9 i+7 \varepsilon+7 h)+(6+4 i+4 \varepsilon+4 h) \\
& =\left(2^{n+m} 385+2^{n} 39+2^{m} 39+6\right)+\left(2^{n+m} 20-2^{n} 9+2^{m} 27+4\right) i
\end{aligned}
$$




$$
+\left(2^{n+m} 40+2^{n} 9+2^{m} 21+4\right) \varepsilon+\left(2^{n+m} 80+2^{n} 33+2^{m} 21+4\right) h
$$

Thus, the proof is obtained.

\section{CONCLUSIONS}

We presented Mersenne-Lucas hybrid numbers. We have given the Binet formula, the generating function, the character and the norm for Mersenne-Lucas hybrid numbers. Also, we have given relations among these numbers. Then we have obtained Cassini identity, Catalan identity, Vajda identity and D'ocagne identity for Mersenne-Lucas hybrid numbers.

\section{ACKNOWLEDGMENTS}

We would like to thank the editor and referees for their valuable comments, and remarks that led to a great improvement of the article.

\section{REFERENCES}

[1] S. Çelik, İ. Durukan, E. Özkan, “New recurrences on Pell numbers, Pell-Lucas numbers, Jacobsthal numbers, and Jacobsthal-Lucas numbers”, Chaos, Solitons \& Fractals, 150, 111173 , (2021)

[2] S. Koparal, N. Ömür, "Some Congruences Involving Catalan, Pell and Fibonacci Numbers", Mathematica Montisnigri, 48, 10-18, (2021).

[3] E. Özkan, "3-Step Fibonacci Sequences in Nilpotent Groups”, Applied Mathematics and Computation, 144, 517-527, (2003).

[4] E. Özkan, "On General Fibonacci Sequences in Groups", Turkish Journal of Mathematics, 27 (4), 525-537, (2003).

[5] E. Özkan, "On truncated Fibonacci sequences", Indian Journal of Pure and Applied Mathematics, 38(4), 241-251 (2007).

[6] E. Özkan, İ. Altun, A.A. Göçer, "On relationship among a new family of k-Fibonacci, k-Lucas numbers, Fibonacci and Lucas numbers”, Chiang Mai J. Sci., 44(4), 1744-1750, (2017).

[7] E. Özkan, A. Aydoğdu, İ. Altun, "Some Identities for A Family of Fibonacci and Lucas Numbers", Journal of Mathematics and Statistical Science, 3(10), 295-303, (2017).

[8] N.Yilmaz, A. Aydoğdu, E. Özkan, "Some properties of $\boldsymbol{k}$-generalized Fibonacci numbers", Mathematica Montisnigri, 50,73-79, (2021).

[9] T. Koshy, Fibonacci and Lucas Numbers with applications, New York: John Wiley and Sons Inc. (2001).

[10] P. Catarino, “'On k-Pell hybrid numbers", Journal of Discrete Mathematical Sciences and Cryptography, 22 (1), (2019).

[11] C. Kızılates, "A new generalization of Fibonacci hybrid and Lucas hybrid numbers", Chaos, Solitons and Fractals, 130, (2020).

[12] N. Kilic, "On k-Jacobsthal and k-Jacobsthal-Lucas hybrid numbers", Journal of Discrete Mathematical Sciences and Cryptography, 24(4), 1063-1074, (2021).

[13] M. Özdemir, "Introduction to hybrid numbers", Advances in Applied Clifford Algebras, 28, (2018). 
[14] A.Szynal-Liana, "The Horadam hybrid numbers", Discussiones Mathematicae-General Algebra and Applications, 38, (2018).

[15] A. Szynal-Liana, I. Wloch, “On Pell and Pell-Lucas hybrid numbers”, Commentationes Mathematicae, 58, (2018) .

[16] A. Szynal-Liana, I. Wloch, "On Jacobsthal and Jacobsthal-Lucas hybrid numbers", Annales Mathematicae Silesianae, 33, (2019).

[17] A. Szynal-Liana, I. Wloch, “'On generalized Mersenne hybrid numbers”, Annales Universitatis Mariae Curie-Sklodowska Lublin-Polonia, 74(1), 77-84, (2020).

[18] D, Taşcı, E. Sevgi, "Some Properties between Mersenne, Jacobsthal and Jacobsthal-Lucas Hybrid Numbers', Chaos, Solitons and Fractals, 146, 110862, (2021).

[19] P. Catarino, H. Campos, P. Vasco, “'On the Mersenne sequence”, CM-Centro de Matemática (2016).

[20] N. Saba, A. Boussayoud, K. V. V. Kanuri, "Mersenne Lucas numbers and complete homogeneous symmetric functions", Journal of mathematics and computer science, 24(2), 127$139,(2021)$.

Received June 5, 2021 